\title{
Apatinib treatment for symptomatic pseudoprogression after standard treatment for glioblastoma multiforme: a case report
}

\author{
Zheng Wang", Fangfang Du\#, Yafang Sun, Yang Guo, Bin Song, Yahong Wang, Xiangyu Shi, \\ Wei Jiang \\ Department of Radiation Oncology, Tianjin Huanhu Hospital, Tianjin 300060, China \\ \#These authors contributed equally to this work. \\ Correspondence to: Wei Jiang, MD. Department of Radiation Oncology, Tianjin Huanhu Hospital, 6 Jizhao Road, Jinnan, Tianjin 300060, China. \\ Email: weijianghh@163.com.
}

\begin{abstract}
Apatinib, a novel tyrosine kinase inhibitor, has anti-angiogenetic effect just as bevacizumab. Although bevacizumab has been used successfully in treating cerebral radiation necrosis, there has yet not any report on that apatinib can treat pseudoprogression with symptoms. Here we report a case of glioblastoma multiforme (GBM) patient with pseudoprogression after receiving the concurrent chemoradiotherapy, which was successfully treated by apatinib. A 51-year-old woman had multiple intracranial lesions (left parietal and right frontal), the primary left parietal lesion was surgically removed and was pathologically confirmed as glioblastoma (WHO grade IV). Then the patient received postoperative temozolomide with concurrent chemoradiotherapy. Three weeks after the radiotherapy, the patient experienced increased intracranial pressure and seizure. Magnetic resonance imaging (MRI) T1 enhancement examination showed an increase of abnormal enhancement range in the area of irradiation. After multiple disciplinary team (MDT) discussion, the patient was diagnosed with pseudoprogression after radiotherapy. Then she was given apatinib for 8 weeks at a dose of $500 \mathrm{mg}$ qd. During the treatment period, the clinical symptoms and corresponding nerve images of the patient have been rapidly improved. In 12 months after the radiotherapy, progression of tumor in the primary site has not been discovered. Apatinib showed a good therapeutic effect and tolerance for the development of pseudoprogression advances with obvious symptoms.
\end{abstract}

Keywords: Apatinib; bevacizumab; glioblastoma; pseudoprogression

Submitted Sep 03, 2019. Accepted for publication Oct 14, 2019.

doi: 10.21037/apm.2019.11.13

View this article at: http://dx.doi.org/10.21037/apm.2019.11.13

\section{Introduction}

Glioblastoma multiforme (GBM) is the most common malignancy of the nervous system in adults. At present, maximally safe surgery alongside postoperative temozolomide with concurrent chemoradiotherapy has become the standard treatment for patients with GBM (1). Although concurrent chemoradiotherapy brings about a curative effect, the incidence of pseudoprogression increases markedly in the target area after radiotherapy. Hoffman et al. first observed and reported the phenomenon of pseudoprogression in 1979 (2). de Wit et al. described the phenomenon in more detail in 2004 (3). However, it was not until 2008 that Taal et al. named this phenomenon "pseudoprogression" for the first time (4).

Bevacizumab, a monoclonal antibody against vascular endothelial growth factor (VEGF), has been successfully used in treating nervous system radiation necrosis (5). Previous studies have confirmed a very similar pathology between radionecrosis and pseudoprogression, which is a specific type of radiation injury (6). Therefore, selectively blocking the pathway of VEGF signaling can be used as an effective treatment method for pseudoprogression. Apatinib (Hengrui Pharmaceutical Co., Ltd., Shanghai, China) is a tyrosine kinase inhibitor that selectively inhibits the VEGF receptor-2 (VEGFR-2), which is a novel VEGF downstream 


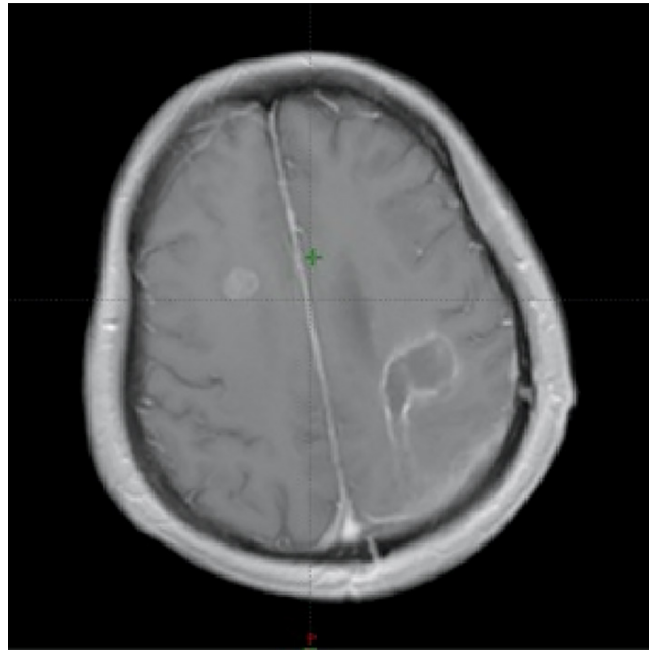

Figure 1 Gd-enhanced T1-weighted MRI postsurgery. MRI, magnetic resonance imaging.

receptor. Apatinib has been approved in China for treating patients with advanced or recurrent gastric adenocarcinoma or gastroesophageal adenocarcinoma who undergo at least two systemic chemotherapy regimens (7). Application of apatinib for treating GBM pseudoprogression after concurrent chemoradiotherapy has not been reported to date. Recently, apatinib was successfully administered to a patient with GBM who had pseudoprogression within a short time after radiotherapy. The details of this case are as follows.

\section{Case presentation}

A 51-year-old female patient presented with rightlimb paralysis as onset. In June 2016, T1-enhanced magnetic resonance imaging (MRI) of the head showed an abnormally enhanced occupancy signal in the left parietal and right frontal areas. Moreover, in July 2016, the left parietal lesion was surgically removed and pathologically confirmed as GBM (World Health Organization, grade IV). A postsurgery Gd-enhanced T1-weighted MRI was performed, as shown in Figure 1. She then received a postoperative 6-week intensity-modulated radiation therapy (IMRT) during which temozolomide $\left[75 \mathrm{mg} / \mathrm{m}^{2}\right.$, once daily (qd)] chemotherapy was synchronized. The IMRT was arranged as follows. In the first phase, radiation was given $2 \mathrm{~cm}$ outside the range of fluid attenuated inversion recovery (FLAIR) abnormal signal at an exposure extent of $40 \mathrm{~Gy} / 20 \mathrm{f}$. This was followed by an MRI re-examination

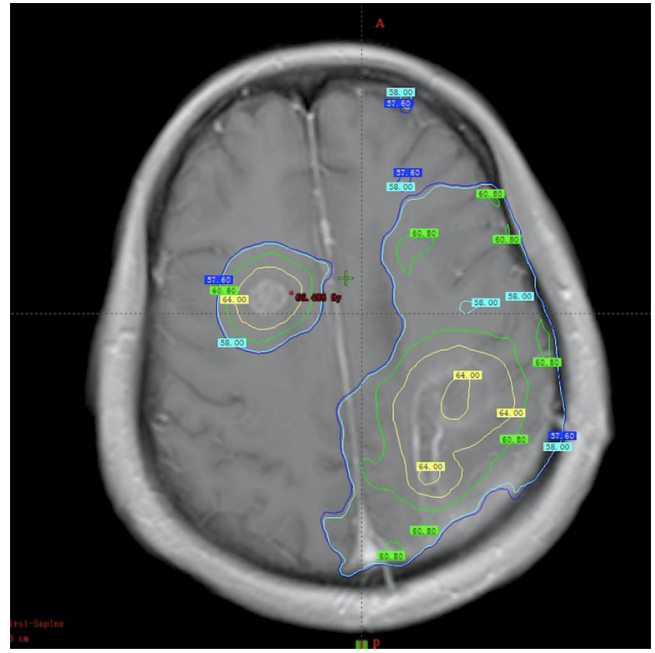

Figure 2 The dose distribution of radiotherapy.

with a simultaneous integrated boost technique conducted for a right frontal lesion in the range where the $\mathrm{T} 1$ was enhanced. Moreover, the left parietal lesions within the surgical cavity were irradiated at $24 \mathrm{~Gy} / 10 \mathrm{f}$, with simultaneous radiation to the area with FLAIR abnormal signal at $18 \mathrm{~Gy} / 10 \mathrm{f}$. The dose distribution is shown in Figure 2. After 3 weeks of concurrent chemoradiotherapy, the patient was admitted to the hospital again due to severe headache and frequent seizures. No significant improvement was observed in the symptomatic treatment therapy of mannitol plus dexamethasone for 1 week. Enhanced T1-enhanced MRI scan showed that the abnormally enhanced range of the original left parietal and right frontal lesions significantly increased compared with that before radiotherapy, as shown in Figure 3. After fusion of the T1-enhanced MRI with the IMRT dose distribution image, the right frontal and left parietal abnormality enhancement signals were found to lay entirely within the 90\% prescription dose field, as shown in Figure 4. The postoperative molecular pathological results showed that the O6-methylguanine-DNA methyltransferase (MGMT) promoter methylation was positive in the left parietal lesion. After a multidisciplinary team discussion, the patient was diagnosed with pseudoprogression after chemoradiotherapy. Continuous temozolomide monotherapy chemotherapy $\left(250 \mathrm{mg} / \mathrm{m}^{2}\right.$ days $\left.1-5 \mathrm{q} 28 \mathrm{~d}\right)$ was given as originally planned due to the poor result of previous dexamethasone treatment. Apatinib, as the concurrent treatment therapy, was given to the patient at a dose of $500 \mathrm{mg}$ qd. During 

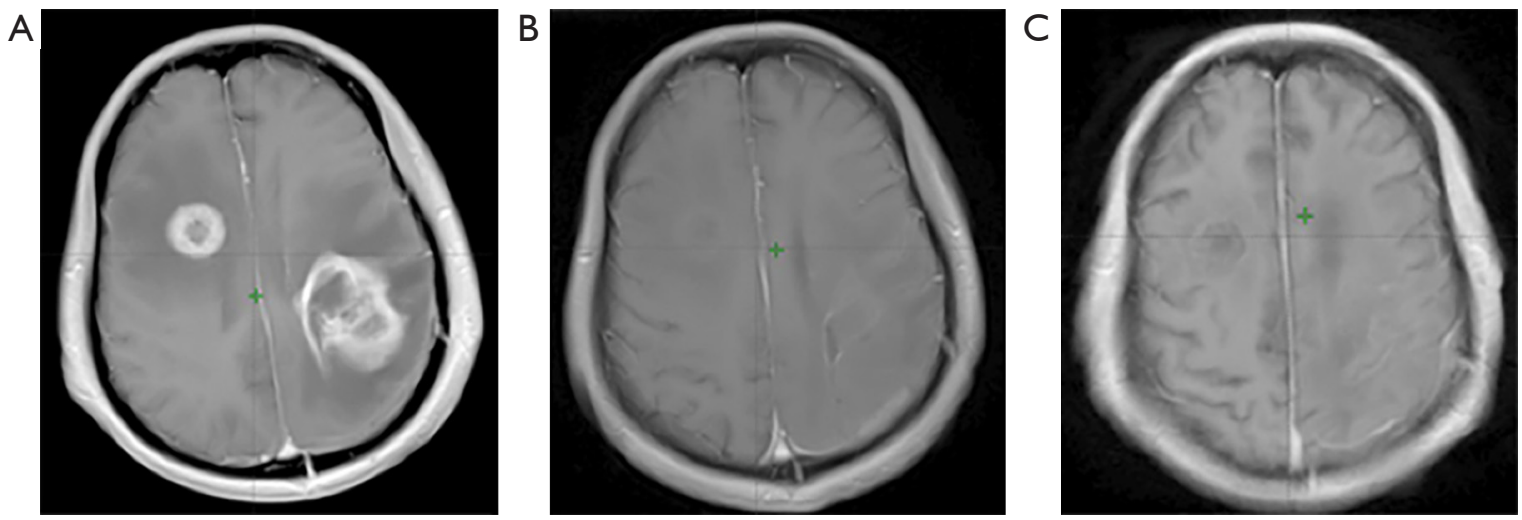

Figure 3 The characteristics of MRI with the patient in different periods. (A) Periodic Gd-enhanced T1-weighted MRI changes with the patient. (B) Gd-enhanced T1-weighted MRI after 1 week of apatinib treatment. (C) Gd-enhanced T1-weighted MRI after 14 months of surgery. MRI, magnetic resonance imaging.

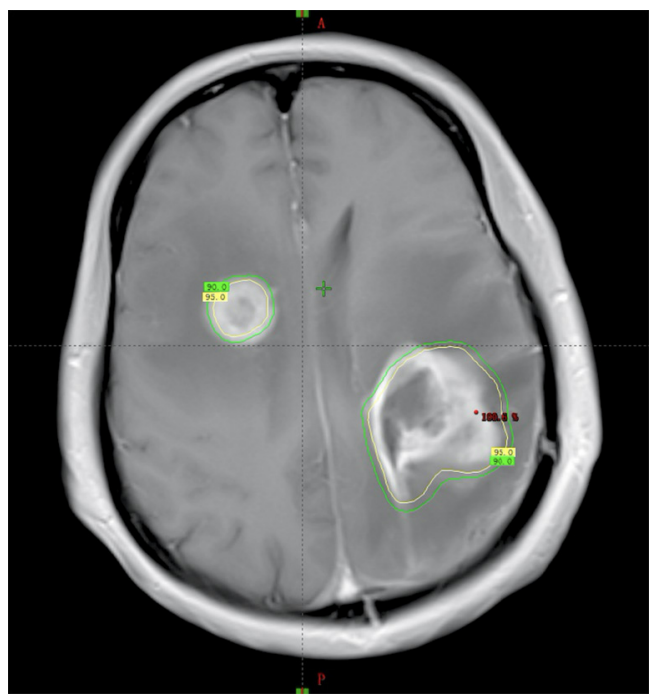

Figure 4 Fusion of the MRI T1 enhancement image with the IMRT dose distribution image. MRI, magnetic resonance imaging; IMRT, intensity-modulated radiation therapy.

this treatment, symptoms of headache and rapid onset of seizures disappeared immediately. After 1 week of treatment with apatinib, MRI examination showed that the scope of the original abnormally enhanced signal was significantly reduced and the peripheral edema was alleviated compared with the images before treatment, as shown in Figure 3. The patient was treated with apatinib (500 $\mathrm{mg} \mathrm{qd}$ ) for 8 weeks, during which the drug was well tolerated and the patient did not show serious adverse effects to apatinib therapy. MRI scan showed no significant abnormally enhanced signal in the area of the primary lesion 14 months after the surgery (in September 2017), supporting the diagnosis of pseudoprogression after initial radiotherapy and chemotherapy, as shown in Figure 3.

\section{Discussion}

The phenomenon of pseudoprogression of tumors after radiotherapy of the nervous system was reported more than 30 years ago. The incidence of pseudoprogression has increased significantly in recent years due to the prevailing application of the Stupp regimen involving temozolomide concurrent chemoradiotherapy (8). According to the latest meta-analysis, $36 \%$ (95\% CI, 33-40\%) of the patients from 73 studies were eventually proven to have pseudoprogression (9). Patients with malignant glioma who received the Stupp regimen had an incidence of pseudoprogression of up to $50 \%(8,10)$. Although the pathological diagnosis is still considered to be the "golden standard" for distinguishing the progression of actual tumor and pseudoprogression, it cannot be widely used because of its invasive operation and a large influence of the site of the tumor. In addition, although functional MRI and other imaging modalities, such as positron emission tomography, can assist the diagnosis of pseudoprogression disease, no universally accepted standard still exists for diagnostic imaging $(8,11)$.

Patients with malignant gliomas with MGMT promoter methylation or isocitrate dehydrogenase (IDH) mutations are more likely to have pseudoprogression after treatment with the Stupp standard regimen $(12,13)$. The reasons may 
result from the previously mentioned two factors, which can lead to an increased sensitivity to radiotherapy and chemotherapy. Brandes et al. retrospectively analyzed data of 103 patients with GBM who underwent adjuvant Stupp regimen. Of these, $32(64 \%)$ of 50 patients who presented with imaging progression in 1 month after surgery were subsequently confirmed with pseudoprogression. Moreover, the incidence of pseudoprogression was $91.3 \%(21 / 23)$ in 23 patients with MGMT-initiated methylation (12). The study by Li et al. had similar conclusions (13).

The diagnosis of pseudoprogression in this study was mainly based on the following features: (I) symptoms appearing in the short term after radiotherapy (within 1 month); (II) an abnormally enhanced signal completely within the scope of prescription dose irradiation; and (III) the molecular pathological examination of postoperative specimens showing MGMT promoter methylation.

"Pseudoprogression" after radiotherapy of the nervous system tumors is considered to be a reversible radioactive injury occurring within the short term after radiotherapy (mostly within 3 months after radiotherapy). However, its specific mechanism is still not clear. Most scholars believe that pseudoprogression has pathological features similar to those of radioactive necrosis. The main manifestations are vascular endothelial damage caused by ionizing radiation, blood vessel barrier occlusion, and small vessel occlusion, further leading to vasogenic brain edema, hypoxia in brain tissues, concomitant VEGF upregulation, increase in vascular permeability, and hypoxia (14). The corresponding imaging features are contrast agent extravasation resulting in the expansion of the scope of enhanced signal and broadening of the peripheral edema zone. The main physiological effect of VEGF on endothelial cells is achieved by activating its receptor VEGFR-2. Therefore, it has been speculated that blocking VEGF/VEGFR-2 signaling pathway can effectively reduce vascular permeability. Apatinib actively competes with VEGFR-2 to attach to the adenosine triphosphate (ATP) binding site in cells. Its corresponding animal test indicated that the inhibitory concentration $\left(\mathrm{IC}_{50}\right)$ for VEGFR-2 was only $2 \mathrm{nM}$ (15). In addition, the oral administration of apatinib is more convenient than the intravenous infusion of bevacizumab. For these reasons, the patient in the present case study was given a lower dose of apatinib (500 mg qd) to treat the radiotherapy-associated pseudoprogression.

Before the advent of anti-angiogenic drugs, corticosteroids were the main treatment for symptomatic pseudoprogression and radiation necrosis. However, their treatment effect is still not satisfactory for some patients and entails additional side effects. In this study, no significant improvement was observed in the symptoms of increased intracranial pressure and frequent seizures within 1 week after treatment with dexamethasone. Fortunately, the patient had significant improvement in her symptoms within 1 week after receiving the treatment with apatinib. Both the range of abnormal enhancement (T1 enhancement) and the edema zone (T2/FLAIR) were narrowed in subsequent imaging examinations. This treatment response was consistent with that reported in previous studies on treatment of radiation necrosis using bevacizumab $(5,16-18)$. Torcuator et al. used bevacizumab to treat 6 patients with pathologically confirmed radiation necrotic lesions and found that the abnormal MRI T1 and FLAIR signal ranges were reduced by $79 \%$ and $49 \%$, respectively, after treatment (17). Sadraei et al. successfully used low-dose bevacizumab to treat 24 patients with radiation necrosis and achieved a reduction of the abnormal MRI T1 range by about $50 \%$ and a narrowing of the abnormal range of FLAIR (18). Interestingly, in this study, an MRI review was performed on the $8^{\text {th }}$ day after the application of apatinib because the patient showed rapid improvement in symptoms. The results showed that the associated enhancement of symptoms and peripheral edema both was significantly improved when compared with those before treatment, indirectly reflecting the high inhibitory effect of apatinib on the VEGF-VEGFR-2 pathway.

In this study, the patient was administered apatinib (500 $\mathrm{mg} \mathrm{qd}$ ) at a lower than usual therapeutic dose. This was because, in addition to the previous experience with bevacizumab, the highly active inhibitory effect of apatinib on the VEGFR-2 target was also considered ( $\mathrm{IC}_{50}$ only $2 \mathrm{nM}$ ) in the present case. Furthermore, apatinib was generally well tolerated by the patient during the treatment process, and she did not experience any serious treatmentrelated side effects until the end of treatment, possibly due to the lower dose of medication.

Apatinib treatment was given for 8 weeks with reference to the application of bevacizumab. During the follow-up of up to 13 months after discontinuation, the patient did not show any pathological changes, or recurrence of the tumor in the original site, or appearance of the aggravation of the original symptoms, supporting the diagnosis of pseudoprogression.

\section{Conclusions}

This study was novel in attempting the application of 
apatinib to a patient with GBM after radiotherapy. The results showed a significant improvement in clinical symptoms and abnormal imaging ( $\mathrm{T} 1 \mathrm{c}+$ and FLAIR). Also, the drug was well tolerated by the patient. Therefore, phase II clinical studies should be conducted to explore the optimal dosage and duration of apatinib in treating pseudoprogression following radiotherapy.

\section{Acknowledgments}

None.

\section{Footnote}

Conflicts of Interest: The authors have no conflicts of interest to declare.

Ethical Statement: The authors are accountable for all aspects of the work in ensuring that questions related to the accuracy or integrity of any part of the work are appropriately investigated and resolved. Written informed consent was obtained from the patient for publication of this manuscript and any accompanying images.

\section{References}

1. Stupp R, Mason WP, van den Bent MJ, et al. Radiotherapy plus concomitant and adjuvant temozolomide for glioblastoma. N Engl J Med 2005;352:987-96.

2. Hoffman WF, Levin VA, Wilson CB. Evaluation of malignant glioma patients during the postirradiation period. J Neurosurg 1979;50:624-8.

3. de Wit MC, de Bruin HG, Eijkenboom W, et al. Immediate post-radiotherapy changes in malignant glioma can mimic tumor progression. Neurology 2004;63:535-7.

4. Taal W, Brandsma D, de Bruin HG, et al. Incidence of early pseudo-progression in a cohort of malignant glioma patients treated with chemoirradiation with temozolomide. Cancer 2008;113:405-10.

5. Levin VA, Bidaut L, Hou P, et al. Randomized doubleblind placebo-controlled trial of bevacizumab therapy for radiation necrosis of the central nervous system. Int J Radiat Oncol Biol Phys 2011;79:1487-95.

6. Nonoguchi N, Miyatake S, Fukumoto M, et al. The distribution of vascular endothelial growth factorproducing cells in clinical radiation necrosis of the brain: pathological consideration of their potential roles. J Neurooncol 2011;105:423-31.

7. Li J, Qin S, Xu J, et al. Randomized, Double-Blind, Placebo-Controlled Phase III Trial of Apatinib in Patients With Chemotherapy-Refractory Advanced or Metastatic Adenocarcinoma of the Stomach or Gastroesophageal Junction. J Clin Oncol 2016;34:1448-54.

8. Nasseri M, Gahramanov S, Netto JP, et al. Evaluation of pseudoprogression in patients with glioblastoma multiforme using dynamic magnetic resonance imaging with ferumoxytol calls RANO criteria into question. Neuro Oncol 2014;16:1146-54.

9. Abbasi AW, Westerlaan HE, Holtman GA, et al. Incidence of Tumour Progression and Pseudoprogression in High-Grade Gliomas: a Systematic Review and MetaAnalysis. Clin Neuroradiol 2018;28:401-11.

10. Brandsma D, Stalpers L, Taal W, et al. Clinical features, mechanisms, and management of pseudoprogression in malignant gliomas. Lancet Oncol 2008;9:453-61.

11. Santra A, Kumar R, Sharma P, et al. F-18 FDG PET$\mathrm{CT}$ in patients with recurrent glioma: comparison with contrast enhanced MRI. Eur J Radiol 2012;81:508-13.

12. Brandes AA, Franceschi E, Tosoni A, et al. MGMT promoter methylation status can predict the incidence and outcome of pseudoprogression after concomitant radiochemotherapy in newly diagnosed glioblastoma patients. J Clin Oncol 2008;26:2192-7.

13. Li H, Li J, Cheng G, et al. IDH mutation and MGMT promoter methylation are associated with the pseudoprogression and improved prognosis of glioblastoma multiforme patients who have undergone concurrent and adjuvant temozolomidebased chemoradiotherapy. Clin Neurol Neurosurg 2016;151:31-6.

14. Nordal RA, Nagy A, Pintilie M, et al. Hypoxia and hypoxia-inducible factor-1 target genes in central nervous system radiation injury: a role for vascular endothelial growth factor. Clin Cancer Res 2004;10:3342-53.

15. Li J, Zhao X, Chen L, et al. Safety and pharmacokinetics of novel selective vascular endothelial growth factor receptor-2 inhibitor YN968D1 in patients with advanced malignancies. BMC Cancer 2010;10:529.

16. Delishaj D, Ursino S, Pasqualetti F, et al. Bevacizumab for the Treatment of Radiation-Induced Cerebral Necrosis: A Systematic Review of the Literature. J Clin Med Res 2017;9:273-80.

17. Torcuator R, Zuniga R, Mohan YS, et al. Initial experience with bevacizumab treatment for biopsy confirmed 
cerebral radiation necrosis. J Neurooncol 2009;94:63-8.

18. Sadraei NH, Dahiya S, Chao ST, et al. Treatment of cerebral radiation necrosis with bevacizumab:

Cite this article as: Wang Z, Du F, Sun Y, Guo Y, Song B, Wang Y, Shi X, Jiang W. Apatinib treatment for symptomatic pseudoprogression after standard treatment for glioblastoma multiforme: a case report. Ann Palliat Med 2019;8(5):752-757. doi: 10.21037/apm.2019.11.13 the Cleveland clinic experience. Am J Clin Oncol 2015;38:304-10. 